\title{
A New Query Method Based on Distance Education Database
}

\author{
Wenxuan $\mathrm{Gu}^{1, \mathrm{a}}$ and Cao Yuan ${ }^{1, \mathrm{~b}^{*}}$ \\ ${ }^{1}$ Mathematic \& Computer Science, Wuhan Polytechnic University, Wuhan, China \\ a6321471958@qq.com, byuancao1980@gmail.com
}

\begin{abstract}
Keywords: Traditional and optimize; Query method; Distance learning; Database
\end{abstract}
\begin{abstract}
In recent years, number of web-based distance learning system has rapidly increased as a result of improvements in the internet. According to the data of different characteristics of the data query optimization will be divided into different categories of data. Through the traditional and optimize two aspects of the distance learning system, using database query way to do an analysis. And I will use the form of the graphic about the study to do a presentation to express my leaning about distance learning database. Typical of distance learning database as follows: query method, the first, database query method based on wheel sequence: From start to finish, then to a circle. And so on, querying a database by this way next time .The second, database query method based on spectral clustering algorithm: The definition of a description of paired data points of similarity affinity matrix, and the calculation of matrix eigenvalue and eigenvector, and then select the appropriate clustering feature vectors of different data points according to the given sample data set. The third, Database query method based on K mean: Given a collection of data points and the number of clustering $\mathrm{K}, \mathrm{K}$ by the user specified, $\mathrm{K}$ mean algorithm based on a distance function repeatedly into k clustering of data.
\end{abstract}

\section{Introduction}

During the development of internet and computer technology, different online learning promotion strategies have different effect on teaching effect [1-2]. The distance education carries numerous advantages, which are related to today's learning ways, the effective study way of distance and study online system. Based on these positive characteristics one can also mention in today's fast adaptation to student study market needs in campus, the rapid innovation of curriculum content, the fast communication with large target groups and their training. Study online in the distance is a very effective form.

Also positive features are that in today's information society, such as internet engineer, database system and other computer technology, changed teacher and student roles, and manage digital citizenship innovative ways.

The new environment in the rapid digital technology, new roles (various students and teachers), attitudes of the digital generation, and the challenges caused by the constant change in the formal education system are trying to be followed, by some study community forums (Innovative Teachers Forum, Digital educators). However, due to the fast and erratic development of multidimensional background influenced by variables the perfect education / learning methodology solutions recipes are clearly not available, although a number of researchers have already showed up results in this area [3-5].

Nowadays, education is not just limited to the classroom and distance learning is becoming more and more popular. Certainly, the development and implementation of new technologies are very important in education. With the development of the information system, the database plays an important role in distance learning [6-7]. The integration of different educational information into a complete database, to provide information for different educational types. Distance learning is a new educational mode; the database also plays a great role in the distance learning mode. The research of data query in database will improve the technology of distance learning. So, the database query method has become a hot research problem in the information management. Pure classroom education can only artificially announced data and query data which is more complex. However, 
Distance learning can query the required data through the database which really improves query quality and speed. There are three ways of data query; I will introduce these methods below. Before I introduce them, we need to learn about some basic knowledge about distance learning system. The means and methods of information transmission in distance learning are different. Distance learning's development experienced three stages:

(1)Exchange information mainly based on mail transfer paper medium.

(2)The teaching stage of the radio by TV broadcast.

(3)The on-line distance learning stage through the computer, the media and the distance communication technology. Nowadays, we develop our distance learning mainly in third ways.

\section{Technologies of Distance Education System}

Database in Distance Education. Database technology focus on the structure of database, storage, design, management, application of basic theory, implementation method. In the distance education system, some researchers make use of these theories to implement process, analyze, and understand technology to apply it in the distance education system.

The important factor is management and research of database in the distance education database. It involved in the specific content mainly includes: management and organization of data, establish the corresponding database and data warehouse based on the specified structure.

In the database system, some function, such as: add, modify, delete, processing, analysis, understanding, report and print can be used that based on a database management system. And the management system can be generated to achieve the data processing, analysis and understanding.

As the distance education is generated based on complicated internet technology, so stronger to database function request, allow data redundancy, needs to be more efficient, and more stable database management system.

Security Technology in Distance Education. Distance education platform involves a large amount of data, the safety of its operation cannot be ignored, must take adequate security measures to ensure the system runs stably and safely. Security technology is introduced as follows:

(1)In term of overall security configuration, mainly on the basis of the existing security technology, increase. Add more security measures, such as installing foreign intrusion detection software, Trojan firewall, etc.

(2)The software security. All users should be logged in when using the network teaching platform.

(3)The data backup and recovery. Timely data should be backup timely, and the backup technology should be controlled strictly, and prevent data corruption and data loss.

(4)The firewall technology. Firewall should have the some functions: ability to filter network number

The Structure of Distance Education System. There are three layers in the structure of distance education system, presentation layer, function layer and database layer as shown in Fig. 1

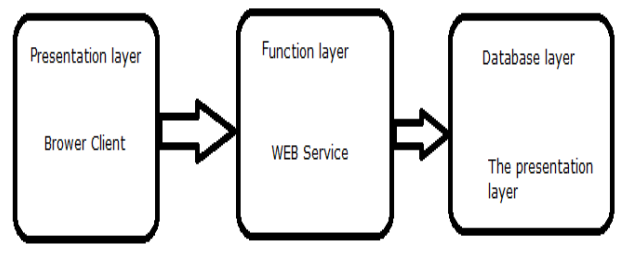

Figure 1. The structure of distance education system

Presentation layer: it is the application of user interface, is responsible for the dialogue between the user and application functions. The input data form key can be checked by the layer, and display the application of the output data source.

Function layer: this layer is the business logic of the application specification, also is the core of the whole system. 
Data layer: mainly be responsible for the management of database data, speaking, reading and writing. In the layer, a large number of data can be updated quickly. Application is more database management system (RDBMS).

\section{Comparsion of Three Query Methods of Database}

Database Query Method Based on Wheel Sequence. Wheel sequence has a simple thought; we sort the required data and regard it as a circle. If left data is needed, this circle can be used as an example and so on. There is no need to explain the first search method with too much explanation.

Database Query Method Based on Spectral Clustering Algorithm. Spectral clustering algorithm, the data set of each object as a graph with vertex $\mathrm{V}$, quantification of the similarity between vertex as vertex of the corresponding connection weights of the edge $\mathrm{E}$, then we get a based on similarity which not has a directed weighted graph $G(V, e)$, and clustering problem can be transformed graph problem. The optimal partitioning criterion based on graph theory is that the similarity between the sub graph and the sub graph is the smallest. Dividing it into three steps:

(1)Construct the similarity matrix of the object set $W$.

(2)The feature vector space is constructed by calculating the similarity matrix or the former $\mathrm{K}$ Eigen values of the Laplace matrix.

(3)Using K-means or other classical clustering algorithm to cluster the feature vector in feature vector space.

Database Query Method Based on K Mean. The K object is randomly selected as the initial clustering center. Then calculate the distance between each object and each seed cluster center, assign each object to its nearest clustering center. The cluster centers and the objects assigned to them represent a clustering. Once all the objects are assigned, each clustering center will be re computed according to the existing object of the clustering [6-7].

\section{A Novel of Optimized Query Methods}

The establishment of the model of multiple constraints is the most important step to optimize the query method. Dividing it into four steps:

Table 1 The sample number of data in the distance learning system

\begin{tabular}{cll}
\hline Attribute ordinal number & \multicolumn{1}{c}{ Sample attribute } & Data quantity \\
\hline 1 & Student A data & 40 \\
2 & Student B data & 40 \\
3 & Student C data & 50 \\
4 & Student D data & 40 \\
5 & Student E data & 50 \\
6 & Teacher A data & 40 \\
7 & Teacher B data & 50 \\
8 & Teacher C data & 50 \\
9 & Teacher D data & 50 \\
10 & lesson A data & 50 \\
11 & lesson B data & 40 \\
\hline
\end{tabular}

The first step: find out the correlation between the data.

The second step: observe the different changes of different data characteristics.

The third step: sum up the similarity between different data.

The last step: the data model is optimized by the establishment of data model.

The sample number of data is 500 in the distance learning system to draw the table 1. 


\section{Conclusion}

From the results of samples in the distance learning system, the condition of the samples can be introduced as fellows:

(1)Same data type : 1, 2, 3, 4, 5 for student ; 6, 7, 8, 9 for teacher ; 10, 11 for lesson. Same amount of data 40 and $50: 1,2,4,6,11$ for $40 ; 3,5,7,8,9,10$ for 50 .

(2)Three data sample: student, teacher and lesson.

(3)All the data are the same source and students can find data from the same direction [8].

(4)Chart classification for optimized data chart classification as shown in Table 2.

Table 2 The table of classification for optimized data in the distance learning system

(1)

\begin{tabular}{clc}
\hline Attribute ordinal number & Sample attribute & Data quantity \\
\hline $1,2,3,4,5$ & Student data & $40,40,50,40,50$ \\
$6,7,8,9$ & Teacher data & $40,50,50,50$ \\
10,11 & Lesson data & 50,40 \\
\hline Attribute ordinal number & Sample attribute & Data \\
\hline $1,2,4,6,11$ & Student,teacher,lesson & $40 * 5$ \\
$3,5,7,8,9,10$ & Student,teacher,lesson & $50 * 6$ \\
\hline
\end{tabular}

In the paper, three query methods of database in distance education system had been compared in the detail; some results can increase the education quality of distance education system in China.

In the summary, the network technology development of distance education resources for students should be used, and the whole process of the teaching process of college students autonomous learning provides a great comfortable convenience, the new query method of database of distance education in modern teaching methods will provoke the enthusiasm of students learning, improve the classroom teaching effect and teaching quality[9].

Nowadays, with the cloud network system, the fully use of modern database, multimedia and network technology, improve the quality of distance education environment and teaching means, and it is the inevitable outcome of the development of a landmark study of information.

Furthermore, university teachers are the most important role of the effective education system, the biggest problems for them is how to face the new network technology application. Teachers should improve their professional quality by studying; learn network application technology, to make the network technology in their class of distance education system [10]. Finally, the improvement of teaching quality can be achieved steadily.

\section{Acknowledgements}

This work and the paper are being supported by the Natural Science Foundation of China (Grant No. 61502362).

\section{References}

[1] G. Eason, B. Noble, and I.N. Sneddon, "On certain integrals of Lipschitz-Hankel type involving products of Bessel functions,” Phil. Trans. Roy. Soc. London, vol. A247, pp. 529-551, April 1955. (references) 
[2] J. Clerk Maxwell, A Treatise on Electricity and Magnetism, 3rd ed., vol. 2. Oxford: Clarendon, 1892, pp.68-73.

[3] I.S. Jacobs and C.P. Bean, "Fine particles, thin films and exchange anisotropy," in Magnetism, vol. III, G.T. Rado and H. Suhl, Eds. New York: Academic, 1963, pp. 271-350.

[4] K. Elissa, "Title of paper if known," unpublished.

[5] R. Nicole, "Title of paper with only first word capitalized," J. Name Stand. Abbrev., in press.

[6] Y. Yorozu, M. Hirano, K. Oka, and Y. Tagawa, "Electron spectroscopy studies on magnetooptical media and plastic substrate interface,” IEEE Transl. J. Magn. Japan, vol. 2, pp. 740-741, August 1987 [Digests 9th Annual Conf. Magnetics Japan, p. 301, 1982].

[7] M. Young, The Technical Writer's Handbook. Mill Valley, CA: University Science, 1989.

[8] Singh. Information System Management[M]. S.B. Nangia APH publishing Corporation. 2011.

[9] Raymond Mcleod,George P.Schell.Management Information Systems[M].Pearson/Prentice Hall, 2007.

[10] Kenneth C.Laudon,Jane Price Laudon.Management Information Systems:Managing the Digital Firm MyMISLab Series[M]. Prentice Hall, 2009. 